\title{
Signal recognition and the probability-matching decision rule
}

\author{
ANGUS CRAIG \\ MRC Perceptual and Cognitive Performance Unit, Laboratory of Experimental Psychology, \\ University of Sussex, Brighton, Sussex, England
}

In a visual binary recognition task in which three levels of signal strength were presented at each of three levels of signal probability, subjects' behavior was consistent with the signal detection theory notion that the sensory and the decision processes are independent, but did not support the contention that the decision processes obey a likelihood ratio rule. Instead, the evidence suggests that subjects were attempting to probability-match, adjusting the relative frequencies of their responses to correspond with those of the stimuli. The results are discussed in relation to previous findings, against the background of signal detection theory.

Experiments on binary discrimination or recognition frequently require an observer to decide which of two signal events, A, B, occurred on a given trial. In these experiments, systematic variations in the observer's response criterion may map out his receiver operating characteristic (ROC), a function which relates the probability of a correct recognition of a signal, $A$ for example [P('A'|A)], to the probability of a false recognition, identifying signal B as signal A[P('A'|B)]. Typically, these functions appear bowed in shape, like those in Figure 1 (e.g., Linker, Moore, \& Galanter, 1964; Tanner, Haller, \& Atkinson, 1967). (Note: Since A and B complement each other, it is simply a matter of personal choice that this entire discussion is in terms of correct and incorrect reports of signal $\mathbf{A}$. It could equally well have been in terms of reports of signal B).

The observer's position on the ROC is determined by his willingness to respond, so that when he is

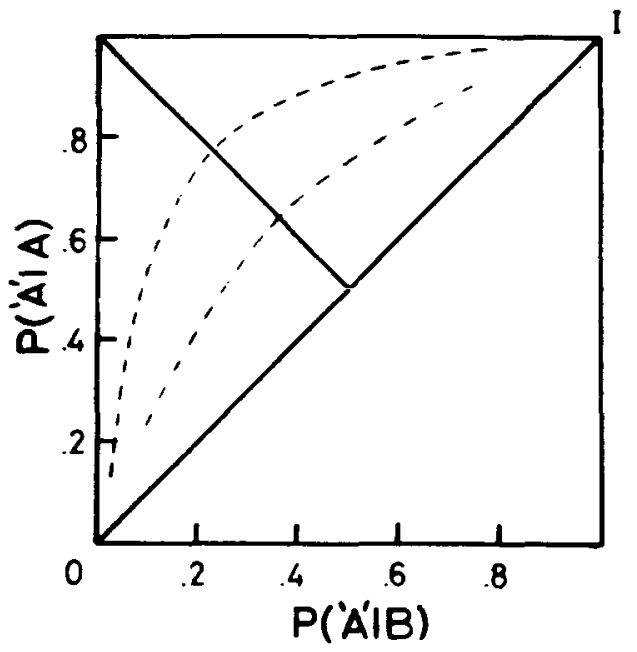

Figure 1. Two typical receiver operating characteristics. being cautious about reporting the presence of signal $A$, or is only willing to report ' $A$ ' when he is very confident about its presence, his behavior places him at the lower left part of the ROC curve. If, on the other hand, he becomes less cautious and reports an A signal, even when he is not at all sure of its presence, then both $P\left({ }^{\prime} A\right.$ ' $\left.\mid A\right)$ and $P\left({ }^{\prime} A\right.$ ' $\left.\mid B\right)$ increase to place him on the upper-right portion of the curve. Galanter and Holman (1967), for example, have shown that the cautiousness of the observer's response set can be altered by verbal instruction and that this manipulation may be used to map out the ROC curve.

The position of an ROC curve within the unit square (as distinct from the operating position on the curve) reflects the discriminability between the signals, and as this increases the whole function shifts upwards and to the left, away from the chance line (OI in Figure 1). A clear example of this effect is contained in the report by Creelman and Donaldson (1968) of a study of line length discrimination.

These described properties of the ROC functions obtained from recognition experiments are consistent with the implications of signal detection theory (Green \& Swets, 1966), which model of performance was originally designated to account for behavior in situations where the observer's task is to detect the presence of a relatively weak signal embedded in a continuous background of random noise.

Signal detection theory (SDT) predicts the general bowed shape of the ROC curves, their shifting upwards and to the left when signal discriminability is increased, and that both correct and false reports increase as the observer's response criterion is relaxed. In terms of these characteristics, therefore, SDT seems just as appropriate for describing recognition behavior as it is for describing behavior in the more fundamental detection situation. 
In addition to the features mentioned, however, SDT also predicts that the observer will alter his response criterion according to the known, a priori probability of signal $A[P(A)]$. At low values of $P(A)$, he will make few ' $A$ ' reports (which corresponds to a cautious criterion), while at high $P(A)$ levels he will make more ' $A$ ' reports of both the correct and the incorrect type. The theory, assuming a rational observer whose goal is either to maximize his percentage of correct responses, or where different values are attributable to correct and incorrect responses, to maximize his net gain, states that optimal behavior would be obtained if he set his criterion at a level where $\beta$, the likelihood ratio of the evidence in favor of signal A's presence, was directly proportional to $[1-P(A)] / P(A)$. In other words, the higher the $P(A)$ level, the less strong the favorable evidence required for the observer to report ' $A$ '.

Whereas the evidence from detection studies is generally consistent with this formulation (Friedman, Carterette, Nakatani, \& Ahumada, 1968; Green \& Swets, 1966), recognition experiments, on the other hand, have produced a miscellany of results, some of which support the SDT model (Baddeley \& Colquhoun, 1969; Linker, Moore \& Galanter, 1964), while others indicate that correct and incorrect report probabilities may actually vary inversely with signal probability (Tanner, Haller, \& Atkinson, 1967).

Although in particular instances one might reasonably suggest that aberrent results could occur because the recognition paradigm violates certain of the SDT assumptions regarding the statistical properties of the stimulus events or because the observer's actual decision goal and his subjective payoff functions are not necessarily those specified by the experimenter, it is clear that no comprehensive account has yet been given for these inconsistent findings.

An associated area of controversy, one which applies to both detection and recognition, concerns the posited relation between $\mathrm{d}^{\prime}$, the discrimination measure, and $\beta$, the criterion index. The formal SDT model states that these two measures are statistically independent and that each is subject to influences which have no correlated effect on the other; that, for example, the strength (S) of signal A (relative to signal B) will influence $d^{\prime}$ in a predictable manner but not $\beta$, while $P(A)$ will induce shifts in the criterion but have no related effect on $\mathrm{d}^{\prime}$. Whether the independence is so in fact as well as in theory is still an unresolved issue.

Most published studies that have manipulated either S or $\mathrm{P}(\mathrm{A})$ have not manipulated the other within the same experiment. If they have used both, the manipulations have usually been kept quite separate. Thus, for example, Linker, Moore, and
Galanter (1964) only varied S with $\mathrm{P}(\mathrm{A})$ constant at one level, and vice versa. Friedman et al. (1968), who manipulated S, P(A), and feedback, used an independent groups design which, of course, precludes the possibility of interactive influences. A few studies, however, have used within-subjects designs which should afford a test of the independence assumptions. Markowitz and Swets (1967), for example, obtain results which appear to show that $P(A)$ does not influence $d^{\prime}$ in a binary response detection task, but that it may do so when observers use multiple rating responses. They do not say whether $S$, which had the predicted effect on $d^{\prime}$, influenced the criterion or not-nor, indeed, do they mention the effects of $P(A)$ on the criterion. Schulman and Greenberg (1970) report findings, also from a ratingmethod detection experiment, indicating that $\mathrm{d}^{\prime}$ will tend to decline as $P(A)$ rises and that increases in $S$ will enhance this effect. Again, they found that values of the discrimination measure increased regularly with increases in $S$ but again, too, they make no comment about effects on the criterion.

The present study attempts to consider this independence issue in more detail and to examine once more the signal probability issue, by looking at the effects of $S$ and $P(A)$ on recognition behavior within the context of a binary response task. In view of the foregoing discussion, the study was designed so that both variables were manipulated within the same experiment, each subject serving under all conditions.

\section{METHOD}

\section{Apparatus}

A visual recognition task was used in which three levels of $P(A)$ were employed at each of three levels of S. The subject's task was to identify the signal present on any trial, responding 'A' or 'B' as appropriate.

The signals consisted of pale green disks, each set in a matt black background and presented behind a mild perspex screen, $15 \mathrm{~cm}$ square, at a viewing distance of $75 \mathrm{~cm}$. Signals were presented at 3-sec intervals for a duration of $1 \mathrm{sec}$. Signal B, which was constant throughout, had an area of $.311 \mathrm{sq} \mathrm{cm}$, while signal A was larger in area by (i) $8 \%$, (ii) $9 \%$, (iii) $11 \%$. The selected values of $P(A)$ were (a) .30 , (b) .50 , (c) .70 . The signals were presented in random sequences with the constraints that the actual $P(A)$ within any given block of 50 trials was within one standard deviation of the stated level, while in total, the actual and stated levels were identical.

\section{Subjects and Procedure}

The subjects were six paid student volunteers aged between 18 and 20 years. None of them had served in any previous experiments and none was studying psychology.

Each subject was tested individually in a 2-h session which consisted of three test periods, one for each level of $S$, with a 5 -min rest interval between each. Sequence was counterbalanced by assigning each subject to one of the six possible permutations of the $\mathrm{S}$ levels. Each period contained three blocks of 150 trials, one block at each of the $P(A)$ levels, with a brief pause 
Table 1

Mean Values of Performance Measures Under Each Experimental Condition

\begin{tabular}{|c|c|c|c|c|c|c|c|c|c|}
\hline & \multicolumn{9}{|c|}{ Strength of Signal A } \\
\hline & \multicolumn{3}{|c|}{$\mathrm{S}_{1}\left(\right.$ Area $\left.=.336 \mathrm{~cm}^{2}\right)$} & \multicolumn{3}{|c|}{$\mathrm{S}_{2}\left(\right.$ Area $\left.=.340 \mathrm{~cm}^{2}\right)$} & \multicolumn{3}{|c|}{$S_{3}\left(\right.$ Area $\left.=.345 \mathrm{~cm}^{2}\right)$} \\
\hline & \multicolumn{3}{|c|}{ Probability of Signal A } & \multicolumn{3}{|c|}{ Probability of Signal A } & \multicolumn{3}{|c|}{ Probability of Signal A } \\
\hline & .3 & .5 & .7 & .3 & .5 & .7 & .3 & .5 & .7 \\
\hline $\begin{array}{l}P(' A \text { ' } \mid A) \\
P\left({ }^{\prime} A \text { ' } \mid B\right) \\
P\left({ }^{s} A '\right) \\
d^{\prime} A \\
\beta\end{array}$ & $\begin{array}{l}.67 \\
.18 \\
.33 \\
1.3 \\
1.39\end{array}$ & $\begin{array}{l}.72 \\
.22 \\
.47 \\
1.4 \\
1.51\end{array}$ & $\begin{array}{l}.76 \\
.26 \\
.62 \\
1.3 \\
1.39\end{array}$ & $\begin{array}{l}.74 \\
.14 \\
.32 \\
1.8 \\
2.01\end{array}$ & $\begin{array}{l}.77 \\
.17 \\
.47 \\
1.7 \\
1.69\end{array}$ & $\begin{array}{l}.79 \\
.18 \\
.61 \\
1.7 \\
1.48\end{array}$ & $\begin{array}{l}.87 \\
.09 \\
.32 \\
2.4 \\
1.41\end{array}$ & $\begin{array}{l}.86 \\
.08 \\
.47 \\
2.2 \\
1.44\end{array}$ & $\begin{array}{c}.83 \\
.07 \\
.61 \\
2.5 \\
.65\end{array}$ \\
\hline
\end{tabular}

of $90 \mathrm{sec}$ between each block. The $\mathrm{P}(\mathrm{A})$ sequences were balanced within and between subjects.

At the beginning of each period and before each block, the subject was shown each disk, first B then A, 12 times and told which was which on each occasion. A random sequence of 24 practice trials was then given, with $P(A)=.50$ and with trial-bytrial feedback on the correctness of the subject's judgments. At this point in the instructions, equal weight was given to the importance of correct recognitions and of avoiding false ones, for both signals. The subject was then informed of the $P(A)$ level to follow, and was told that this was an average value only. He was not told how many trials would be given. Twenty warm-up trials were then presented, followed without pause by the test block. No feedback was given during the testing.

\section{RESULTS}

The mean values of all measures obtained are presented in Table 1.

\section{Signal Strength (S)}

Analyses of variance showed that both correct and false recognitions of signal A were influenced by $S$, its relative strength. As $S$ increased so that the two signals became more disparate, $\mathbf{P}\left({ }^{\prime} \mathbf{A}\right.$ ' $\left.\mid \mathbf{A}\right)$ increased $[F(2,10)=17.389, p<.001]$ while $P(' A$ ' $\mid B)$ declined $[F(2,10)=5.403, p<.05]$. This is the expected "shift upwards and to the left" which was mentioned previously, and it is reflected in the significant effect of $S$ on $d^{\prime}[F(2,10)=23.44$, $\mathrm{p}<.001]$. There is no evidence that $\mathrm{S}$ had any effect on $\beta$, the criterion index $(\mathrm{p}>.20)$. Nor did it have a significant effect on the overall probability of an 'A' report, $\mathrm{P}$ ('A'), which may also be considered as a measure of response criterion.

\section{Signal Probability [P(A)]}

There was no significant effect of $P(A)$ on the probability of either a correct or an incorrect ' $A$ ' report. This in itself suggests that signal probability did not have the predicted effect on the subjects' criterion, a surmise which is borne out by the analysis of variance of the $\beta$ measures. The obtained $F$ ratio of 1.529 corresponds to a significance level of $p>.20$.

There was, however, a striking effect of $P(A)$ on the overall probability of an 'A' report $[F(2,10)=$
115.476, $\mathrm{p}<.001]$, as Figure 2 illustrates. The linear component of the $\mathrm{P}(\mathrm{A})$ sum of squares was highly significant $\left[F_{\text {lin }}(1,10)=230.10, p<.001\right]$; in fact, it accounted for $94.3 \%$ of the total sum of squares.

There was no effect of $P(A)$ on $d^{\prime}(F=.108)$ and the results show no indication of interaction effects.

\section{DISCUSSION}

The results confirm the existence of two independent processes in recognition. On the one hand, there is a process which reflects the sensitivity of the observer to changes in relative signal strength and which
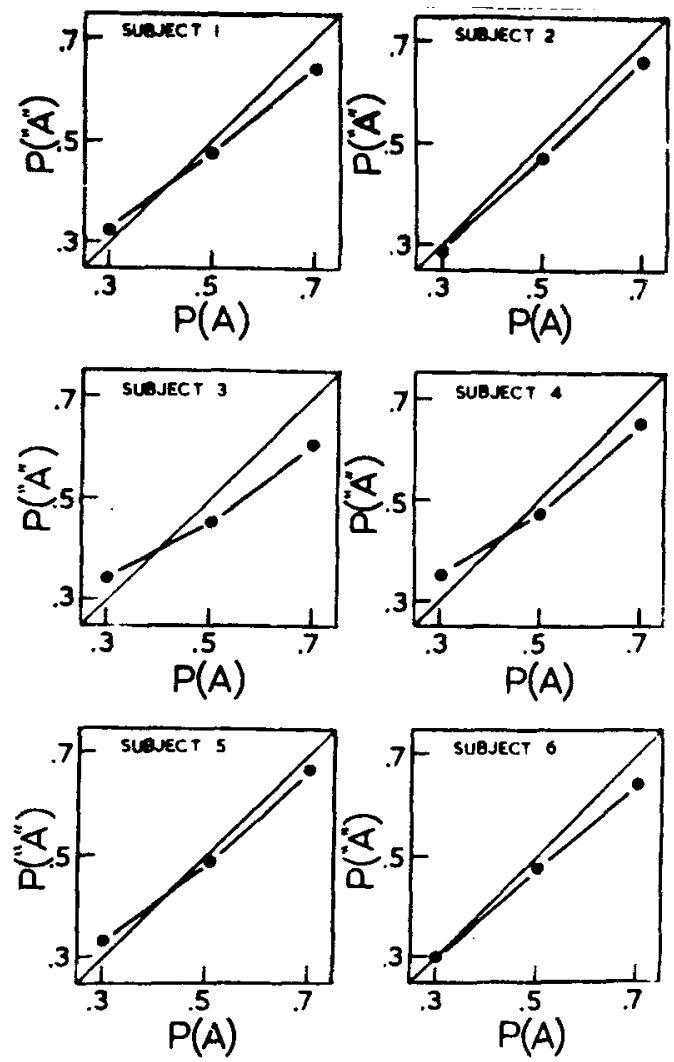

Figure 2. Individual probability-matching functions. 
is expressed here in terms of the d' measure; on the other hand, it seems that the P('A') measure reflects some form of decision process, whereby the observer alters his criterion for responding ' $A$ ' according to the relative frequency of $A$ signals being presented. No statistically significant relation was found between the measures in the sense that there were no observable changes in $d^{\prime}$ as signal probability was altered, nor in $\mathbf{P}$ ('A') as $\mathbf{S}$ was increased. The failure to observe any significant interactions in the analyses of variance further suggests that $P(A)$ and $S$ influence processes which are not statistically related.

The lack of association between $\mathrm{d}^{\prime}$ and signal probability corroborates the findings of Markowitz and Swets (1967) in their binary response condition, but it conflicts with those of Schulman and Greenberg (1970), who used the rating method. The latter authors made the observation that $\mathrm{d}^{\prime}$ would tend to fall as signal probability was increased. However, calculations based on the values they report, suggest that $\mathrm{d}^{\prime}$ would fall by only about 0.10 for a rise in $P(A)$ of 0.20 , and that this would not have reached significance, had they tested for it. It seems fair to state, therefore, that the evidence favors the theoretical notion of an independent sensory process in detection and in recognition.

What then of the response criterion? The SDT model predicts that the index $\beta$ will vary inversely with signal probability, with "optimal" values defined by $\beta(o p t)=(1-p) / p$, assuming a symmetric payoff function as was specified in the instructions to the subjects. In the present study, where $P(A)$ was raised from .30 to $.70, \beta$, according to this model, should have fallen from a value greater than 2.0 to a value less than 1.0. In fact, this did not happen. There was no apparent shift in $\beta$, the median values for $P(A) s$ of $.30, .50$, and .70 being $1.23,1.33$, and 1.29 respectively. These results cast serious doubts on the predictive value of the model, not only because they fail to agree with the specific predictions of the equation given, but also because the model contains sufficient flexibility to account for them. For instance, one could speculate that the subjective payoff function of the observer did not remain stationary and symmetrical as $P(A)$ was altered, but shifted in such a way that the net expected gain from making an ' $A$ ' or $a$ ' $B$ ' report varied inversely with the relative frequency of the signal; that, for example, when there were few A events, the value or importance of a correct identification was high, but declined as the $\mathrm{A}$ events became more frequent, while the value of correctly identifying $B$ events increased. If the observer were trying to maximize his overall gain, these conjectured variations in payoffs could have effectively damped the expected shifts in $\beta$ (see Green \& Swets, 1966, pp. 22-23). That such unfounded suppositions can, with some propriety, be invoked to account for a failure in prediction is seen as a weakness as well as a strength of the SDT model.

A more supportable inference is that the observers were not using the posited likelihood-ratio rule at all. Thus, the failure to observe a shift in $\beta$ does not imply that they were not altering the criterion in a regular observable manner as signal probability was varied. On the contrary, the $P\left({ }^{\prime} A\right.$ ') results suggest that they were. The reported relation between $P(' A$ ') and the probability of signal $A$ suggests a decision strategy whereby the relative frequency of response ' $A$ ' is altered so that it mirrors the relative frequency of signal $A$.

The observation that subjects were probabilitymatching while their $\beta$ values remained constant is at variance with the SDT model. The notion of probability-matching as an alternative rationale for setting decision criteria was advanced by Atkinson (1963) and has been observed subsequently in several studies (Atkinson, Carterette, \& Kinchla, 1964; Creelman \& Donaldson, 1968; Kinchla \& Atkinson, 1964; Parks \& Kellicut, 1968; Kinchla, Note 1). As Creelman and Donaldson (1968) have shown, when observers are using the matching strategy, $\beta$ remains close to a value of 1.0 over the middle range of $P(A)$ values, especially when monetary payoffs are low. The present study confirms this.

The probability-matching interpretation, however, is not congruent with the result noted earlier, that neither the probability of a correct recognition nor the probability of a false recognition was affected by $P(A)$.

For the probability-matching rule, iso-bias functions, the curves joining points where the criterion or response-bias has a constant value, are straight lines of negative slope, radiating from the upper left corner of the unit square within which the ROC lies (see Appendix). This rule, combined with an ROC curve of monotonically decreasing slope [as is consistent with the finding that the $d^{\prime}$ statistic did not change as $P(A)$ was varied], is illustrated in Figure $3 \mathrm{~A}$, from which it is obvious that both the
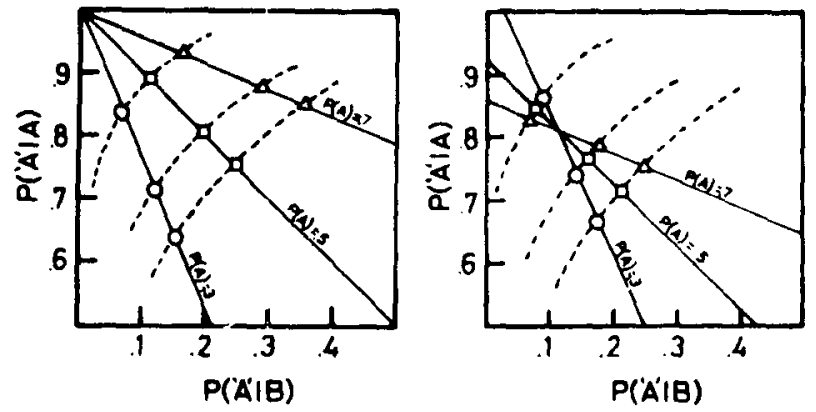

Figure 3. (a) Idealized and (b) observed operating character. istics and isobias functions. 
correct and incorrect recognition probabilities should increase with increases in $P(A)$. Figure 3B illustrates why this was not observed in the present study. When the probability-matching is not perfect, the iso-bias lines intersect at a single point which, as here, may be within the unit square (see Appendix), and when an ROC intersects the lines above this point, the relation between the recognition probabilities and $P(A)$ is reversed. The net effect is therefore to reduce or even cancel out the effect of $P(A)$. Figure 3 might also explain why certain authors have found either no relation or a negative one between signal probability and P('A'). (Parducci \& Sandusky, 1965, 1970; Tanner, Haller, \& Atkinson, 1967; Tanner, Rauk, \& Atkinson, 1970).

In conclusion, therefore, it appears from the present results that recognition behavior does indeed involve two independent processes, a sensory discrimination mechanism, which is influenced by physical attributes of the stimuli, and a decision process, sensitive to changes in the presentation probabilities. The precise nature of the latter process remains unspecified, but it does seem to involve a response-bias function whose parameter is itself a linear function of signal probability.

\section{APPENDIX}

\section{Isobias Functions Corresponding to the Probability-Matching Rule}

The present findings indicate that $\mathbf{P}\left({ }^{\prime} \mathrm{A}^{\prime}\right)$ and $\mathbf{P}(\mathbf{A})$ are linearly related. If it is assumed that this is true in general, then the relation may be expressed simply as:

$$
P\left({ }^{\prime}\right)=m P(A)+c,
$$

where $m$ and $c$ are constants. This may be equated with the identity:

$$
P\left({ }^{\prime} A^{\prime}\right)=P\left({ }^{\prime} A^{\prime} \mid A\right) \cdot P(A)+P\left({ }^{\prime} A^{\prime} \mid B\right)[1-P(A)]
$$

to yield:

$$
\mathbf{P}\left({ }^{\prime} A^{\prime} \mid A\right) \cdot P(A)+P\left({ }^{\prime} A^{\prime} \mid B\right)[1-P(A)]=m P(A)+c
$$

Dividing both sides of Equation (2) by $P(A)$ :

$$
\begin{aligned}
& P\left({ }^{\prime} A^{\prime} \mid A\right)+P\left(A^{\prime} \mid B\right)[1-P(A)] / P(A)=m+c / P(A), \\
& \text { i.e., } \\
& P\left({ }^{\prime} A^{\prime} \mid A\right)=-P\left({ }^{\prime} A^{\prime} \mid B\right)[1-P(A)] / P(A)+m+c / P(A)
\end{aligned}
$$

This is the equation of a straight line relating $\mathrm{P}\left({ }^{\prime} \mathrm{A}\right.$ ' $\left.\mid \mathrm{A}\right)$ and $\mathrm{P}\left({ }^{\prime} \mathrm{A}\right.$ '| $\left.\mathrm{B}\right)$, with $\mathrm{P}(\mathrm{A})$ as parameter. The line is of negative slope for $\mathrm{O}<\mathrm{P}(\mathrm{A})<1.0$. Since all points on the line will also satisfy the matching rule expressed in Equation 1, Equation 3 can be regarded as the isobias function.

Consider, now, any two values of $P(A), p$ and $q$, say. The corresponding isobias functions will have unequal slopes and so must intersect. The point of intersection can be found by solving the simultaneous equations:

$$
\begin{aligned}
& P\left({ }^{\prime} A^{\prime} \mid A\right)=-P\left({ }^{\prime} A^{\prime} \mid B\right)(1-p) / p+m+c / p \\
& P\left({ }^{\prime} A^{\prime} \mid A\right)=-P\left({ }^{\prime} A^{\prime} \mid B\right)(1-q) / q+m+c / q
\end{aligned}
$$

The solution is:

$$
P\left({ }^{\prime} A^{\prime} \mid A\right)=m+c ; P\left({ }^{\prime} A^{\prime} \mid B\right)=c .
$$

Since $p, q$ were any values of $P(A)$, the solution will hold for all values of $P(A)$ within the stated range $\mathrm{O}<\mathrm{P}(\mathrm{A})<1.0$.

Thus, all isobias functions defined by Equation 3 will intersect at the single point $(c, m+c)$. This point will lie within the unit square of the receiver operating space for $O<c<1$ and $O<m+c<1$.

In the special case of perfect probability matching $\left[P\left({ }^{\prime} A^{\prime}\right)=P(A)\right]$, the values of $m$ and $c$ will be 1.0 and 0 , respectively, so that the isobias functions will intersect at the point $(0,1)$, the upper left corner of the unit square.

\section{REFERENCE NOTE}

1. Kinchla, R. A. A comparison of sequential effects in detection and recognition. Experimental Psychology Series, Psychology Department, New York University. Technical Report Number 1, 1966.

\section{REFERENCES}

Atkinson, R. C. A variable sensitivity theory of signal detection. Psychological Review, 1963, 70, 91-106.

Atkinson. R. C., Carterette, E. C., \& Kinchla, R. A. The effect of information feedback upon paychophysical judgments. Psychonomic Science, 1964, 1, 83.

Baddeley, A. D., \& Colouroun, W. P. Signal probability and vigilance: A reappraisal of the 'signal-rate' effect. British Journal of Psychology, 1969, 60, 169-178.

Creelman, C. D., \& Donaldson, W. ROC curves for discrimination of linear extent. Joumal of Experimental Psychology, $1968,77,514.516$.

Friedman, M. P., Carterette, E. C., Nakatani, L., \& Ahumada, A. Comparisons of some learning models for response bias in signal detection. Perception \& Psychophysics, $1968,3,5-11$.

Galanter, E., \& Holman, G. L. Some invariances of the isosensitivity function and their implications for the utility of money. Joumal of Experimental Psychology, 1967, 73, 333-339.

Green, D. M., \& Swets, J. A. Signal detection theory and psychophysics. New York: Wiley, 1966.

Kinchla, R. A., \& Atkinson, R. C. The effect of false information feedback upon psychophysical judgments. Psychonomic Science, $1964,1,317$.

Linker, E., Moore, M. E., \& Galanter, E. Taste thresholds. 
detection models and disparate results. Joumal of Experimental Psychology, 1964, 67, 59-66.

Markowitz, J., \& SwETS, J. A. Factors affecting the slope of empirical ROC curves: Comparison of binary and rating responses. Perception \& Psychophysics, 1967, 2, 91-101.

Parducci, A., \& Sandusky, A. Distribution and sequence effects in judgment. Journal of Experimental Psychology, 196, 69, 450-459.

Parducci. A.. \& Sandusky, A. Limits on the applicability of signal detection theories. Perception \& Psychophysics, 1970, 7 , 63-64.

Parks. T. E., \& Kellicutt, M. H. The probability-matching rule in the visual discrimination of order. Perception \& Psychophysics. $1968,3,356-360$.
Schulman, A. I.. \& Greenberg. G. Z. Operating characteristics and a priori probability of the signal. Perception \& Psychophysics, 1970, 8, 317-320.

Tanner. T. A.. Haller, R. W., \& Atrinson, R. C. Signal recognition as influenced by presentation schedules. Perception \& Psychophysics, 1967, 2, 349-358.

Tanner, T. A.. Rauk, J. A., \& Atkinson, R. C. Signal recognition as influenced by information feedback. Journal of Mathematical Psychology, 1970, 7. 259-274.

(Received for publication June 11, 1975; revision received March 4, 1976.) 